\title{
Extraction of material parameters in 1-D fractal metamaterial
}

\author{
Samaneh Moeini* and José Carlos Pedro** \\ Instituto de Telecomunicações, Departamento de Eletrónica, Telecomunicações e Informática, Universidade de Aveiro, \\ 3810-193 Aveiro, Portugal
}

Received 15 April 2016 / Accepted 19 June 2016

\begin{abstract}
In this paper, we develop a transfer matrix-based homogenization approach applicable to self-similar fractal structures. With this method, we derive the material parameters of a layered metamaterial comprising two different dielectrics with permittivities equal to $\varepsilon_{1}$ and $\varepsilon_{2}$, respectively, in which the layers are distributed based on a fractal set. The band dispersion diagram and effective permittivity obtained analytically are verified with numerical simulations.
\end{abstract}

Key words: Metamaterials, Fractals, Material parameters.

\section{Introduction to the problem}

The complexity of metamaterials (MTMs) [1] gives rise to various homogenization techniques, which allow for an efficient analytical description of their electromagnetic properties without the need to consider all of their structural details [2-7].

The majority of the known homogenization methods for MTMs rely on the periodicity of the structure. Although the spatial period in such MTMs is typically much smaller than the wavelength, it is always finite, and thus a finite-size MTM sample always contains a finite number of unit cells.

In this work, we are interested in fractal [8] MTMs, constituents of which follow a fractal downscaling rule and therefore have (theoretically) infinitely many internal parts of infinitesimal dimensions. Here, the self-similarity properties of fractals are exploited for proposing a new homogenization method for such MTMs based on fractal geometry.

As a test problem for this homogenization method, we consider a layered fractal MTM comprising two different dielectrics. In this case, an unbounded fractal MTM can be formed by periodically repeating fractal unit cells. In the following we consider a structure having just one such cell. Figure 1 depicts examples of the unit cells with increasing fractal order. In the first order (Figure 1a), we consider three layers: one layer of $\varepsilon_{1}$ with the thickness of $d_{1}$ in the middle and two layers with permittivity $\varepsilon_{2}$ and the thickness of $d_{2}$. The structure is along the $z$ axis and has a total thickness of $L=d_{1}+2 d_{2}$. The layers are considered infinite along $y$ and $x$ axes (as compared to the wavelength).

By increasing the fractal order, the first and third layers of the first-order structure are replaced by a triplet of layers which

\footnotetext{
*e-mail: samaneh@ua.pt

**e-mail: jcpedro@ua.pt
}

are self-similar to the original structure, but with the roles of $\varepsilon_{1}$ and $\varepsilon_{2}$ interchanged. For a fractal of $n$-th order this procedure is repeated $n-1$ times. As an example, the first four resulting profiles $\varepsilon(z)$ are shown in Figure 1.

The geometry of the fractal structure is determined by one parameter: the fractal ratio, $r$, which is defined as $\frac{d_{2}}{L}$ and thus by definition can not be higher than 0.5 .

\section{Analytical transfer matrix-based approach}

The effective parameters of the 1D fractal medium can be calculated analytically using the transfer (ABCD) matrix approach. The transfer matrix, $A$, is defined as follows [9]:

$$
\left(\begin{array}{l}
E_{x 1} \\
H_{y_{1}}
\end{array}\right)=A_{\text {diel }} \cdot\left(\begin{array}{l}
E_{x 2} \\
H_{y_{2}}
\end{array}\right),
$$

in which $E_{x 1,2}$ and $H_{y_{1,2}}$ are the transverse fields at the input and the output of the structure and $A_{\text {diel }}$ is the transfer matrix of a dielectric layer which can be written as shown below [10]:

$$
A_{\text {diel }}=\left(\begin{array}{cc}
\cos k d & j \eta \sin k d \\
\frac{j}{\eta} \sin k d & \cos k d
\end{array}\right),
$$

where $d$ is the thickness of the dielectric layer, and

$$
\begin{gathered}
\eta=\eta_{0} \sqrt{\frac{\mu_{\mathrm{r}}}{\varepsilon_{\mathrm{r}}}}, \\
k=k_{0} \sqrt{\varepsilon_{\mathrm{r}} \mu_{\mathrm{r}}},
\end{gathered}
$$

and $\eta_{0}$ and $k_{0}$ are the wave impedance and the wave propagation factor in free space, respectively. 
(a)

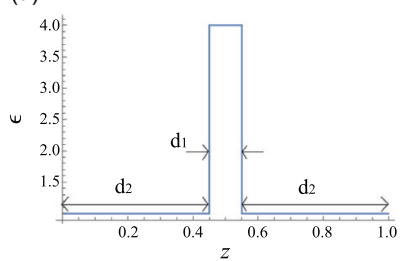

(c)

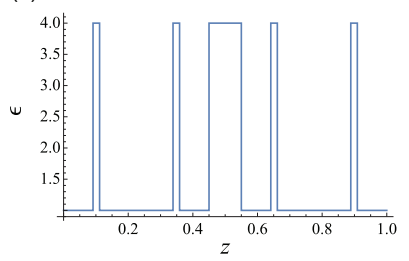

(b)

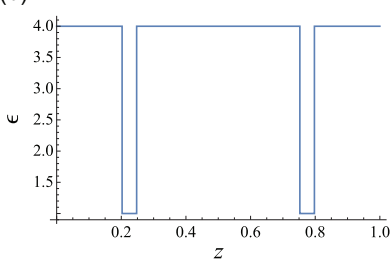

(d)

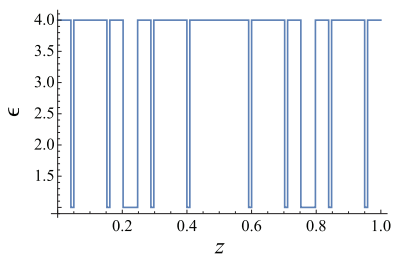

Figure 1. $\varepsilon$ profile for different orders of fractal MTM. In this example, $L=d_{1}+2 \times d_{2}=1, d_{2}=0.45 L, \varepsilon_{1}=4$ and $\varepsilon_{2}=1$. (a) 1 st order, (b) 2 nd order, (c) 3rd order, (d) 4th order.

For nonmagnetic materials, we have $\mu_{\mathrm{r}}=1$ and if we use the field units such that $\eta_{0}=1$, the equation for $A_{\text {diel }}$ with relative permittivity $\varepsilon_{\mathrm{r}}$ can be simplified as shown below:

$$
A_{\text {diel }}=\left(\begin{array}{cc}
\cos k_{0} \sqrt{\varepsilon_{\mathrm{r}}} d & j \frac{\sin k_{0} \sqrt{\varepsilon_{\mathrm{r}}} d}{\sqrt{\varepsilon_{\mathrm{r}}}} \\
j \sqrt{\varepsilon_{\mathrm{r}}} \sin k_{0} \sqrt{\varepsilon_{\mathrm{r}}} d & \cos k_{0} \sqrt{\varepsilon_{\mathrm{r}}} d
\end{array}\right) .
$$

Using these expressions, the total transfer matrix of a stack of dielectric layers is calculated as an ordered product of the transfer matrices of the separate layers.

\subsection{Eigenvalues of the transfer matrix and the band dispersion diagram}

By knowing the eigenvalues of the transfer matrix, the band diagram [11, p. 29] of the structure can be calculated. The eigenvalues $\Lambda_{1,2}$ of the total transfer matrix are related to the propagation factors $k_{z}=k_{0} \sqrt{\varepsilon_{\mathrm{eff}}}$ as $\Lambda_{1,2}=\exp ^{ \pm j k_{z} L}$. Within propagation bands, $k_{z}$ is real (when there is no loss) and the two eigenvalues of the transfer matrix are complex conjugate of each other. On the other hand, within stopbands, $k_{z}$ is purely imaginary and the two eigenvalues have distinct real values.

The band diagram (the dispersion characteristic) for the fractal structure of 9th order obtained with the transfer matrix approach is shown in Figure 2. In this diagram, the propagation factor $k_{z}$ is calculated as:

$$
k_{z}=\frac{1}{L}\left|\operatorname{Im}\left(\log \Lambda_{1}\right)\right| .
$$

\subsection{Effective permittivity in the quasi-static approximation}

When the fractal order is increasing, the total number of layers in the structure grows very fast (in geometric

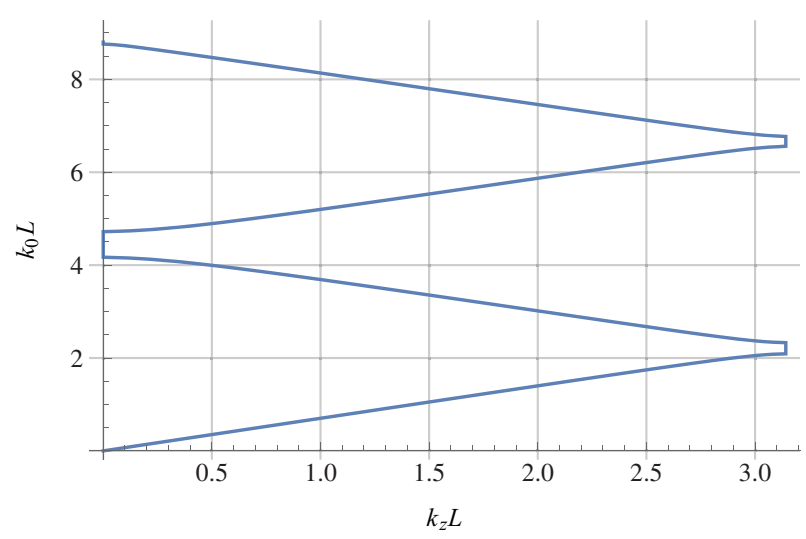

Figure 2. The band diagram for the 9th order fractal MTM. Four propagation bands and three band gaps are seen in the diagram.

progression), which makes direct numerical computation of the total transfer matrix and the band diagram inefficient. Therefore, to describe fractal structures of order $n \rightarrow \infty$, an alternative approach is needed.

In order to develop such approach, we note that the transfer matrix of the whole structure of infinite fractal order $A_{\infty}$ can be calculated recursively due to the self-repeating property of the fractal:

$$
A_{\infty}\left(k_{0}, L, \varepsilon_{1}, \varepsilon_{2}\right)=B_{\infty} \cdot A_{\text {diel }}\left(k_{0}, d_{1}, \varepsilon_{1}\right) \cdot B_{\infty},
$$

where $B_{\infty}$ is the transfer matrix of the two fractal parts which surround the middle dielectric layer of thickness $d_{1}=(1-2 r) L$. By using fractal's self-similarity property, $B_{\infty}$ can be expressed as

$$
\begin{aligned}
B_{\infty}= & A_{\infty}\left(k_{0}, r^{2} L, \varepsilon_{1}, \varepsilon_{2}\right) \cdot A_{\text {diel }}\left(k_{0}, r d_{1}, \varepsilon_{2}\right) \\
& \cdot A_{\infty}\left(k_{0}, r^{2} L, \varepsilon_{1}, \varepsilon_{2}\right)
\end{aligned}
$$

The equations (7) and (8) are functional equations for the unknowns $A_{\infty}$ and $B_{\infty}$. Resolving these equations analytically for arbitrary values of the parameters appears impossible. However, when interested only in the behaviour of the structure at low frequencies, the following method can be used. The same approach also allows us to find the effective permittivities of the structure at low frequencies.

In order to solve equations (7) and (8) with the effective medium approach, we equate the unknown transfer matrices $A_{\infty}$ and $B_{\infty}$ to the transfer matrices of uniform dielectric layers with some effective permittivities $\varepsilon_{A \text {,eff }}$ and $\varepsilon_{B \text {,eff: }}$ $A_{\infty}=A_{\text {diel }}\left(k_{0}, L, \varepsilon_{A \text {,eff }}\right)$ and $B_{\infty}=A_{\text {diel }}\left(k_{0}, r L, \varepsilon_{B, \text { eff }}\right)$, and search for such $\varepsilon_{A \text {,eff }}$ and $\varepsilon_{B \text {,eff }}$ which satisfy the equations (7) and (8). In the quasi-static limit of $k_{0} L \ll 1$ the expression for the transfer matrix of the dielectric layer can be approximated as:

$$
A_{\text {diel }}\left(k_{0}, d, \varepsilon\right)=\left(\begin{array}{cc}
1-\frac{\varepsilon}{2}\left(k_{0} d\right)^{2} & \mathrm{i} k_{0} d \\
\mathrm{i} \varepsilon k_{0} d & 1-\frac{\varepsilon}{2}\left(k_{0} d\right)^{2}
\end{array}\right)+\mathrm{O}\left(\left(k_{0} d\right)^{3}\right) .
$$


Using this expression for $A_{\infty}$ and $B_{\infty}$, we solve the system of equations (7) and (8). The solution of this system of equations is:

$$
\begin{aligned}
& \varepsilon_{A, \mathrm{eff}}=\frac{\varepsilon_{1}+2 \varepsilon_{2} r}{1+2 r}, \\
& \varepsilon_{B, \mathrm{eff}}=\frac{\varepsilon_{2}+2 \varepsilon_{1} r}{1+2 r},
\end{aligned}
$$

where, $\varepsilon_{A \text {,eff }} \equiv \varepsilon_{\text {eff }}$ is the effective epsilon of the whole fractal. Note that, in the quasi-static limit, the effective permittivity of the structure is expressed as a weighted average of the permittivities of the layers.

In order to understand the range of applicability of the obtained quasi-static expressions for the effective permittivity, we compare the eigenvalues of the total transfer matrices calculated by the effective medium approach and by the multilayer approach.

The transfer matrix eigenvalues for both the effective medium approach and the direct numerical calculation for multiple layers are shown in Figure 3. This figure depicts the real part and the absolute value of the imaginary part of the two eigenvalues as functions of $k_{0} L$. The curves representing these functions (the blue and golden curves) coincide within the propagation bands and split (form "loops" visible in Figure 3 ) in the stopbands. Figure 3 shows that outside of the stopbands and when $k_{0} L \lesssim 2$, the result from the effective medium calculations is similar to the result from numerical calculations for 9 th order.

\section{FDTD simulations}

In order to verify the analytical results obtained with the transfer matrix approach, MIT Photonic-Bands $(\mathrm{MPB})^{1}$ package and $\mathrm{MEEP}^{2}$ simulation software package have been used [12].

In contrast with the transfer-matrix based calculations, in which considering structures that have thousands of dielectric layers is feasible, in FDTD-based numerical calculations working with higher orders of fractals is more difficult. Therefore, the following results are given only for 9th and 11th order fractal MTMs which have 683 and 2,731 dielectric layers in the whole structure, respectively. Figure 4 shows the $\varepsilon$ profile of the fractal of 9th order.

\subsection{FDTD-based band diagram calculations}

Figure 5a depicts the permittivity profile of the 9th order fractal MTM as it is represented by the MPB. With increasing

\footnotetext{
${ }^{1}$ The MIT Photonic-Bands (MPB) package is a free program for computing the band structures (dispersion relations) and electromagnetic modes of periodic dielectric structures.

${ }^{2}$ Meep (or MEEP) is a free finite-difference time-domain (FDTD) simulation software package developed at MIT to model electromagnetic systems, along with MPB eigenmode package.
}

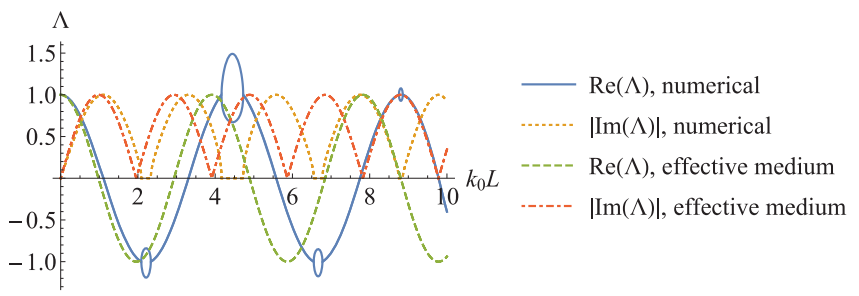

Figure 3. The imaginary and real parts of eigenvalues obtained from numerical results and effective medium calculations for the 9th order fractal MTM.

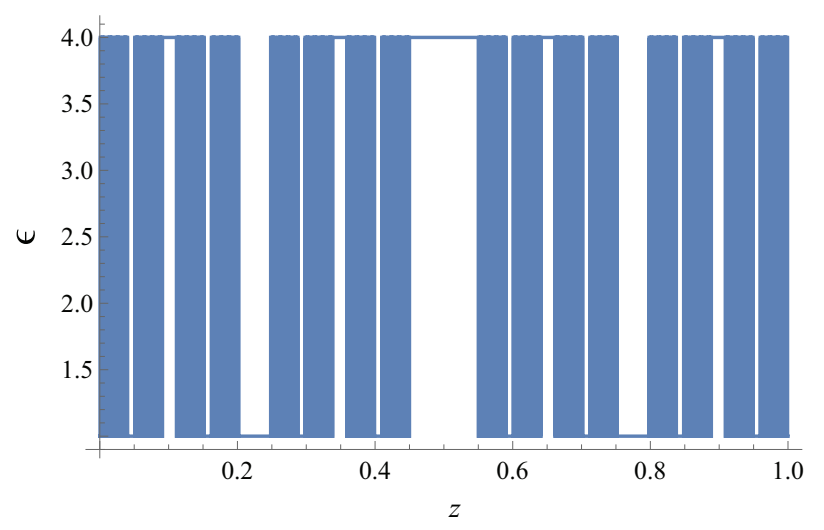

Figure 4. $\varepsilon$ profile for the 9 th order fractal MTM. Due to the limited resolution of the figure, the very thin dielectric layers located in the colored regions are not seen.

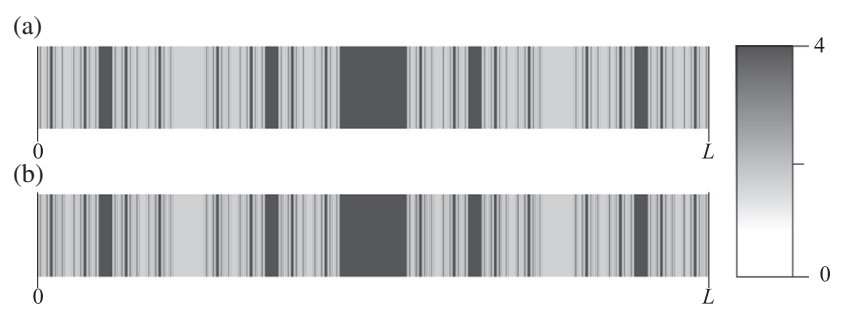

Figure 5. $\varepsilon$ profile for the 9 th and 11 th order fractal MTM by MPB. The black and gray color are $\varepsilon_{1}$ and $\varepsilon_{2}$ respectively. (a) 9th order, (b) 11 th order.

the resolution of the figure the distribution of $\varepsilon$ is better seen for higher orders.

The number of layers in each order of this structure is $\frac{1}{3}\left(2^{n+2}-(-1)^{n}\right)$. The smallest layer thickness equals to $L r^{n}$ for $\frac{1}{3}<r<\frac{1}{2}$, and $L(1-2 r) r^{n-1}$ for $r<\frac{1}{3}$. Considering these conditions, the physical limits for the highest order of this structure can be estimated.

Figure 6 shows the band diagram for the 9th and 11th orders (the epsilon profile of this order is shown in Figure $5 b$ ) of fractal MTM. As is seen from the band diagram for 


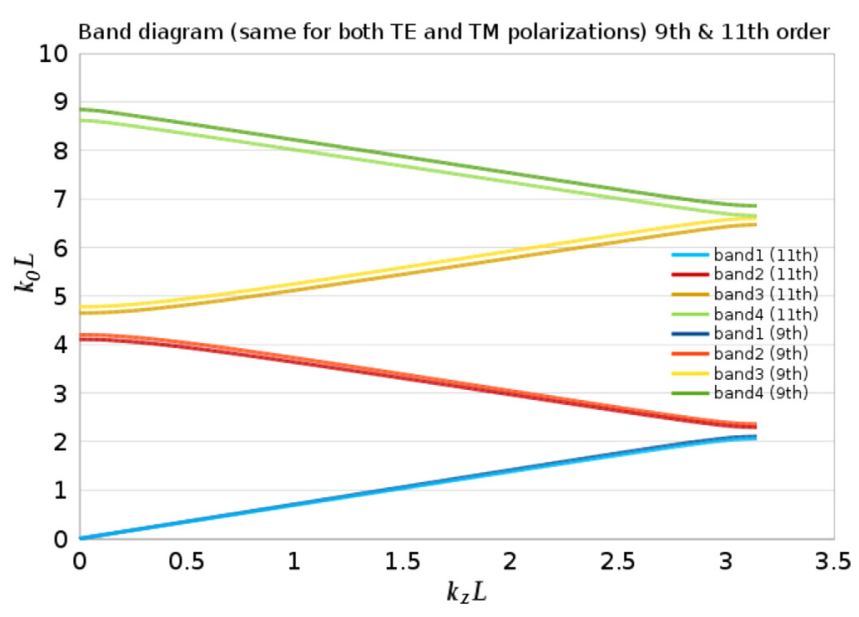

Figure 6. Band diagrams for 9 th and 11 th order by MPB.

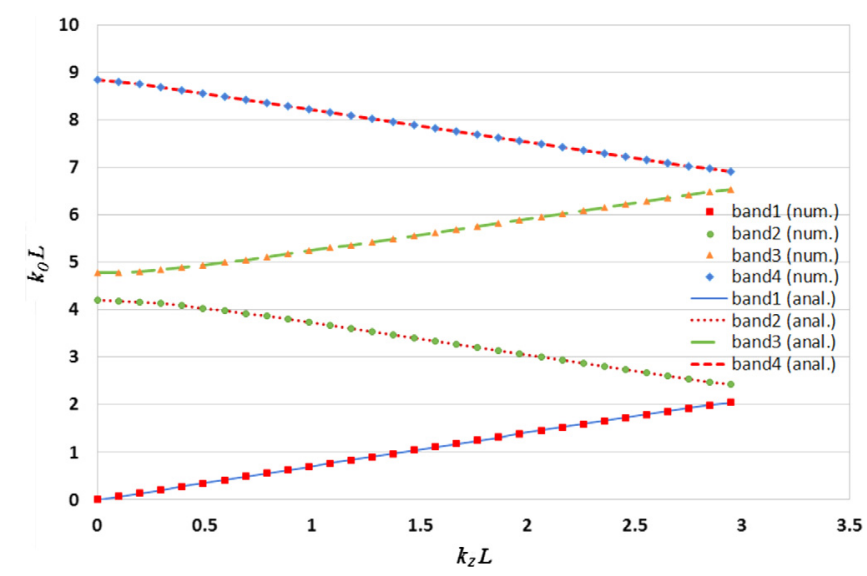

Figure 7. Comparison between band diagrams from analytical and numerical calculation for a 9th order fractal MTM.

the 9th order, the simulation results follow closely the analytical results shown in Figure 2.

As it is seen from Figure 6, since the finest fractal elements are already too small in these orders (as compared to wavelength), the band diagrams are very close to each other.

Figure 7 shows the agreement between analytical results and numerical calculations for the fractal of 9th order.

\subsection{Field distributions in the propagation bands}

Using the FDTD algorithm allows us also to study how the electromagnetic field of the modes propagating inside the structure is distributed within the multilayer structure.

The field distribution along the fractal structure is shown in Figure 8, for four bands of propagation. In these examples, $k_{z} L=\frac{\pi}{2}$. As one can see, the field distributions closely resemble the ones for plane waves propagating in uniform media, which shows that the studied fractal structure can be considered as effectively homogeneous in these frequency bands.

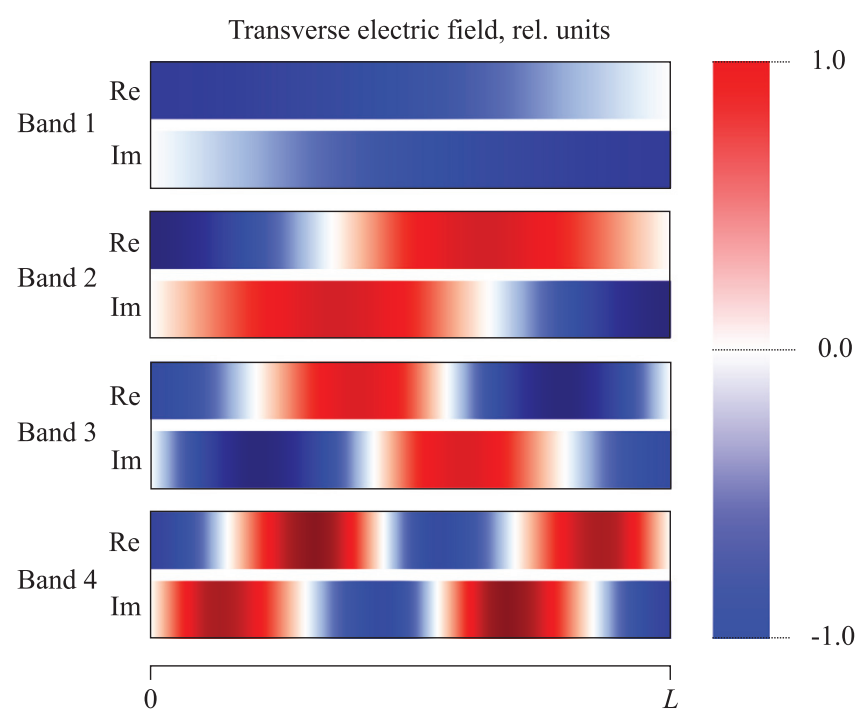

Figure 8. Distribution of the transverse electric field (real and imaginary parts) inside the fractal structure for different propagation bands (in units relative to the field maximum).

\section{Conclusion}

In this work a new 1D metamaterial which is based on a fractal set has been presented. A homogenization method that uses self-similarity property of the structure has been developed. We have derived the effective material parameters of this fractal MTM structure and verified them numerically. The quasi-static effective permittivity obtained with this method is expressed as a weighted average of permittivity of dielectric layers in the fractal. This result agrees well with the known quasi-static solutions for layered media, which confirms the validity of the proposed homogenization method.

Acknowledgements. This work, is funded by FCT/MEC through national funds (PD/BD/52589/2014) and when applicable co-funded by FEDER PT2020 partnership agreement under the project UID/ EEA/50008/2013.

\section{References}

1. V.G. Veselago, Sov. Phys. Usp. 10 (1968) 509.

2. J.V. Kranendonk, J. Sipe, Progress in Optics 15 (1977) 245-350, DOI: 10.1016/S0079-6638(08)70480-3.

3. D.R. Smith, J.B. Pendry, J. Opt. Soc. Am. B 23 (3) (2006) 391, DOI: $10.1364 / J O S A B .23 .000391$.

4. O. Ouchetto, C.W. Qiu, S. Zouhdi, L.W. Li, A. Razek, IEEE Trans. Microwave Theory Tech. 54 (2006) 3893, DOI: 10.1109/ TMTT.2006.885082.

5. C.R. Simovski, Opt. Spectrosc. 107 (2009) 726, DOI: 10.1134/ S0030400X09110101.

6. E. Poutrina, D. Huang, D.R. Smith, New J. Phys. 12 (2010) 093010.

7. A. Alù, Phys. Rev. B 84 (2011) 075153, DOI: 10.1103/ PhysRevB.84.075153. 
8. K. Falconer, Fractal Geometry: Mathematical Foundations and Applications, 3rd edn., John Wiley \& Sons, Inc, UK, 2014.

9. D.M. Pozar, Microwave Engineering, 4th edn., John Wiley \& Sons, Inc, UK, 2011, https://books.google.pt/books?id= JegbAAAAQBAJ.

10. S. Tretyakov, Analytical Modeling in Applied Electromagnetics, Artech House, USA, 2002.
11. J.D. Joannopoulos, S.G. Johnson, J.N. Winn, R.D. Meade, Photonic Crystals, 2nd edn., Princeton University Press, USA, 2008, http://press.princeton.edu/titles/8696.html.

12. A.F. Oskooi, D. Roundy, M. Ibanescu, P. Bermel, J. Joannopoulos, S.G. Johnson, Comput. Phys. Commun. 181 (2010) 687, DOI: 10.1016/j.cpc.2009.11.008.

Cite this article as: Moeini S \& Pedro JC: Extraction of material parameters in 1-D fractal metamaterial. EPJ Appl. Metamat. $2016,3,7$. 